\title{
ENFERMAGEM E METAS INTERNACIONAIS DE SEGURANÇA: AVALIAÇÃO EM HEMODIÁLISE
}

\author{
Letícia Lima Aguiarr, Maria Vilani Cavalcante Guedes², Roberta Meneses Oliveira ${ }^{3}$, Ilse Maria Tigre de \\ Arruda Leitão ${ }^{4}$, Viviane Peixoto dos Santos Pennafort ${ }^{5}$, Ariane Alves Barros ${ }^{6}$
}

\begin{abstract}
RESUMO: Objetivou-se descrever o cuidado de enfermagem prestado em serviço hospitalar de hemodiálise com base em metas internacionais de segurança do paciente. Estudo descritivo realizado no serviço de hemodiálise de hospital público brasileiro, referência no atendimento de pacientes renais agudos e crônicos, entre agosto e outubro de 2013. Participaram 25 profissionais de enfermagem. Os dados foram coletados com entrevista, observação e checklist baseados nas metas de segurança da Joint Commission International. Observou-se que as metas em conformidade foram: comunicação efetiva; redução do risco de infecções associadas aos cuidados de saúde; e redução do risco de lesões decorrentes de quedas. As demais metas, que são: identificação correta do paciente; segurança no manuseio de medicamentos de alta vigilância; e assegurar procedimento em local correto, merecem atenção por parte da equipe estudada e pela instituição avaliada. Constatou-se a necessidade de implementação de protocolos no serviço para a segurança do paciente e da equipe.

DESCRITORES: Enfermagem; Segurança do paciente, Enfermagem em nefrologia; Unidades hospitalares de hemodiálise.

\section{NURSING AND INTERNATIONAL SAFETY GOALS: HEMODIALYSIS ASSESSMENT}

ABSTRACT: The objective of this study was to describe the nursing care offered at a hemodialysis hospital service based on international patient safety goals. A descriptive study was conducted at the hemodialysis service of a Brazilian public hospital, reference in care for acute and chronic kidney patients, between August and October, 2013. Twenty-five nursing professionals participated in the study. Data were collected through interviews, observation and a checklist based on the safety goals of the Joint Commission International. The goals found to be in conformity were: improving effective communication; reducing the risk of healthcare-associated infections; and reducing the risk of patient harm resulting from falls. The remaining goals, namely: identifying patients correctly; improving the safety of high-alert medications; and ensuring safe surgery, deserve attention from the researched team and the assessed institution. The research found a need for the implementation of protocols in the service aimed at patient and team safety. DESCRIPTORS: Nursing; Patient Safety, Nephrology Nursing; Hospital Hemodialysis Units.
\end{abstract}

\section{ENFERMERÍA Y METAS INTERNACIONALES DE SEGURIDAD: EVALUACIÓN EN HEMODIÁLISIS}

RESUMEN: Se objetivó describir la atención de enfermería brindada en servicio hospitalario de hemodiálisis en base a metas internacionales de seguridad del paciente. Estudio descriptivo realizado en servicio de hemodiálisis de hospital público brasileño, referencia en atención de pacientes renales agudos y crónicos, entre agosto y octubre de 2013. Participaron 25 profesionales de enfermería. Datos recolectados mediante entrevista, observación y checklist basados en metas de seguridad de la Joint Commission International. Las metas en conformidad fueron: comunicación efectiva; reducción del riesgo de infecciones asociadas a cuidados de salud; y reducción del riesgo de lesiones provocadas por caídas. Las demás metas, identificación correcta del paciente; seguridad en manipulación de medicamentos de alta vigilancia; y asegurar procedimientos en ubicación correcta, merecen atención del equipo estudiado y de la institución evaluada. Se constató necesidad de implementación de protocolos en el servicio para seguridad del paciente y del equipo.

DESCRIPTORES: Enfermería; Seguridad del Paciente; Enfermería en Nefrología; Unidades de Hemodiálisis en Hospital.

${ }^{1}$ Enfermeira. Doutoranda em Enfermagem. Universidade Federal do Ceará. Fortaleza, CE, Brasil.

${ }^{2}$ Enfermeira. Docente do Programa de Pós-Graduação em Cuidados Clínicos em Enfermagem e Saúde e Graduação da Universidade Estadual do Ceará. Fortaleza, CE, Brasil.

${ }^{3}$ Enfermeira. Doutora em Cuidados Clínicos em Enfermagem e Saúde. Docente de Enfermagem da Universidade Federal do Ceará. Fortaleza, CE, Brasil.

${ }^{4}$ Enfermeira. Doutora em Saúde Coletiva. Docente da Universidade Estadual do Ceará. Fortaleza, CE, Brasil.

${ }^{5}$ Enfermeira. Doutora em Cuidados Clínicos em Enfermagem e Saúde. Enfermeira Nefrologista do Hospital Universitário Onofre Lopes. Natal, RN, Brasil.

${ }^{6}$ Enfermeira. Doutoranda em Cuidados Clínicos em Enfermagem e Saúde. Universidade Estadual do Ceará. Fortaleza, CE, Brasil.

Autor Correspondente:

Recebido: 01/03/2016

Letícia Lima Aguiar

Finalizado: 06/07/2017

Universidade Federal do Ceará

R. Sátiro Dias, 522 - 60420-430 - Fortaleza, CE, Brasil

E-mail: leticiaaguiar1991@gmail.com 


\section{INTRODUÇÃO}

Apesar dos avanços na área da segurança do paciente, o erro humano, entendido como falha na execução de um plano de ação, é um fator que se destaca nas instituições hospitalares. O erro pode acarretar, para o profissional envolvido, sentimentos de vergonha, culpa e medo, dada a forte cultura punitiva ainda existente nas instituições, contribuindo para a omissão dos episódios ${ }^{(1)}$.

Essa realidade tem chamado a atenção dos pesquisadores e sociedade em geral, considerando a crescente divulgação de eventos adversos hospitalares. Sendo assim, a segurança do paciente tornouse pauta inserida nas políticas de saúde em todo o mundo ${ }^{(2)}$.

$\mathrm{Na}$ atenção a pacientes renais crônicos, a realização do tratamento dialítico tem aumentado nas últimas décadas. Segundo a Sociedade Brasileira de Nefrologia, o número de pacientes em diálise passou de 42.695 em 2000 para 112.682 no ano de $2015^{(3)}$. Assim, cresce a preocupação com eventos adversos nessa área, pois estes pacientes têm maior vulnerabilidade a incidentes ${ }^{(4-6)}$.

Para promover segurança e proteção ao paciente durante a sessão de hemodiálise (HD), enfermeiro e equipe de enfermagem devem usar técnica asséptica e prevenir a infecção ao cuidar desse paciente; avaliar constantemente os resultados individuais dos pacientes; monitorizar sinais vitais; observar manifestações corporais como: dor, empatia, sorriso, afeto, atenção; avaliar a ultrafiltração e atentar para sinais de intercorrências intradialíticas ${ }^{(7)}$.

A introdução das metas de segurança do paciente em serviço hospitalar de hemodiálise é uma forma de minimizar pontos que não atendam aos padrões internacionais e nacionais. Para tal, pode-se fazer uso das ferramentas de acreditação hospitalar ${ }^{(8)}$.

A Organização Mundial de Saúde (OMS) criou um grupo de trabalho para disseminar a cultura de segurança do paciente nos serviços de saúde em 2004, intitulada Aliança Mundial para a Segurança do Paciente ${ }^{(9)}$. No Brasil, em 2013, a Portaria Ministerial 529/2013 instituiu o Programa Nacional de Segurança do Paciente (PNSP), que contribui para a qualificação do cuidado em saúde em todos os estabelecimentos de saúde do território nacional ${ }^{(10)}$.

No país, há três instituições atuando como acreditadoras no Brasil: a Organização Nacional de Acreditação; a Comissão Conjunta Internacional (Joint Commission International - JCI), representada pelo Consórcio Brasileiro de Acreditação; e o Conselho Canadense de Acreditação de Serviços de Saúde (Canadian Council on Health Services Accreditation - CCHSA), representada pelo Instituto Qualisa de Gestão(11).

A Joint Commission International (JCI), por meio da avaliação das Metas Internacionais de Segurança do Paciente, visa reduzir erros decorrentes da assistência à saúde em ambientes de cuidado ${ }^{(12)}$. Tais metas são: 1 - Identificar os Pacientes Corretamente; 2 - Melhorar a Comunicação Efetiva; 3 - Melhorar a Segurança de Medicamentos de Alta Vigilância; 4 - Assegurar Cirurgias com Local de Intervenção Correto, Procedimento Correto e Paciente Correto; 5 - Reduzir o Risco de Infecções Associadas aos Cuidados de Saúde; 6 - Reduzir o Risco de Lesões ao Paciente Decorrentes de Quedas ${ }^{(12)}$.

Este estudo objetivou descrever o cuidado de enfermagem prestado em serviço hospitalar de hemodiálise com base em metas internacionais de segurança do paciente.

\section{MÉTODO}

Pesquisa descritiva, realizada em uma unidade de referência em hemodiálise de um hospital público de Fortaleza-Ceará-Brasil, de agosto a outubro de 2013.

O serviço dispõe de 19 máquinas de hemodiálise e 20 poltronas elétricas. Ao todo, são 12 máquinas funcionando em regime de 24 horas, atendendo a pacientes em unidades de terapia intensiva, transplante renal e sala de recuperação, estabilização e emergência do hospital. A equipe interdisciplinar do setor é formada 76 profissionais.

A equipe de enfermagem desta unidade é composta por 57 trabalhadores de enfermagem, sendo 
15 enfermeiros e 42 técnicos.

Participaram da pesquisa 25 trabalhadores de enfermagem: sete enfermeiras e 18 técnicas de enfermagem, que trabalhavam na unidade no período diurno há pelo menos um ano. Excluíram-se quem se encontrava afastado por férias ou licença.

Os dados foram coletados por meio de entrevista semiestruturada e um checklist de observação sistemática elaborado pelas pesquisadoras, baseado nas metas de segurança do paciente da Joint Commission $^{(12)}$.

O roteiro de entrevista abordava: a dinâmica do serviço; o tratamento hemodialítico e seus riscos; percepção sobre eventos adversos; e os cuidados realizados junto aos pacientes, com enfoque na segurança.

Por sua vez, o checklist foi criado com base em estudo(6) que descreve eventos adversos relatados por profissionais de enfermagem de uma Unidade de Hemodiálise. Este incluía informações sobre a frequência de realização e registros do observador relacionados a: Passagem de plantão e suas características; Planejamento do cuidado; Registros; Intercorrências clínicas e incidentes com o paciente; e Medidas para proteção do paciente/boas práticas. Foi utilizado diário de campo para o registro de aspectos não contemplados nos demais instrumentos de coleta.

Os dados das entrevistas foram transcritos, lidos e tratados conforme a Análise de Conteúdo Temática $^{(13)}$. Garantiu-se a confidencialidade dos dados e o anonimato dos profissionais, que foram identificados com a letra E quando enfermeiros, e TE quando técnicos de enfermagem, seguidos de numeral arábico conforme a ordem em que foram entrevistados.

A apresentação dos resultados foi realizada de acordo com a descrição dos dados colhidos com os diferentes instrumentos e a análise de sua conformidade com as metas internacionais de segurança do paciente. Desse modo, buscaram-se as inter-relações do processo de hemodiálise com a segurança do paciente observada e registrada pelas pesquisadoras ${ }^{(12)}$.

A pesquisa foi aprovada em agosto de 2013 pelo Comitê de Ética em Pesquisa (Parecer $n^{\circ}$ 376.199/13). Durante a coleta de dados, foram observadas as diretrizes e os critérios éticos de pesquisa junto a seres humanos, de acordo com a Resolução nº 466 de 12 de dezembro de 2012 do Conselho Nacional de Saúde (CNS) ${ }^{(14)}$.

\section{- RESULTADOS}

A amostra foi formada por mulheres, com idade média de 41 anos $( \pm 7,9)$, tempo médio de formação na área de Enfermagem de nove anos $( \pm 6,0)$, tempo médio de atuação no serviço de hemodiálise de quatro anos $( \pm 4,9) ; 40 \%$ trabalhava mais de 40 horas semanais; todas as enfermeiras eram especialistas em Nefrologia.

Sobre as metas internacionais de segurança do paciente avaliadas, observou-se melhor desenvoltura no atendimento dos aspectos relacionados às metas 2, 5 e 6 . Por outro lado, observaram-se falhas nas demais metas, apresentadas a seguir.

Na Meta 1 foi possível constatar que apenas o nome do paciente, e por vezes, a prescrição eram conferidos antes do procedimento iniciar. No entanto, havia um impresso ao lado de cada máquina, que identificava o paciente a ser submetido à hemodiálise e seus respectivos níveis pressóricos e peso. Além disso, não eram adotadas pulseiras de identificação.

Algumas entrevistadas manifestaram preocupação diante da identificação correta do paciente a ser submetido à hemodiálise:

[...] tem que observar o paciente, se é o paciente certo que vai ser ligado, se é o setor certo em relação ao leito [...]. (E3)

Antes de ligar o paciente na máquina, confiro se eu estou ligando o paciente certo, se a prescrição que eu tenho na mão bate com o nome dele, é basicamente isso. (TE12) 
Na Meta 2 percebeu-se que o processo de comunicação apresentou lacunas importantes, como: não participação das enfermeiras e dos médicos nefrologistas na visita aos pacientes que dialisavam nas áreas externas e a falta de padronização ao transmitir informações ao telefone sobre o estado geral e o quadro clínico dos pacientes a serem submetidos ao procedimento.

A enfermeira responsável pelas diálises externas recebia informações e comunicava-se por telefone com a enfermeira do setor onde o paciente estava internado. Porém, em discurso abaixo, uma enfermeira deixou claro que esse contato acontece apenas quando surge uma intercorrência.

[...] a gente liga para o setor para perguntar as condições hemodinâmicas do paciente,[...]então a gente encaminha a técnica de enfermagem para fazer a diálise. Caso aconteça uma intercorrência, a gente mantém contato com a enfermeira do setor, só na existência de um evento que impeça que a diálise flua normalmente, caso contrário, a gente não tem esse contato "durante" a diálise [...]. (E5)

Observou-se que não havia a repetição, pelo receptor, da mensagem recebida por telefone, o que pode gerar falhas na comunicação e riscos à segurança do paciente.

Já na área interna da unidade de diálise, o processo de comunicação era mais efetivo, com enfoque na confirmação de resultados de exames laboratoriais e na leitura de registros nos prontuários. A enfermeira que atuava diretamente nesta unidade discutia o caso do paciente com o médico e colhia informações relevantes que pudessem comprometer a diálise do paciente, conforme se confirma no seguinte depoimento:

[...] o enfermeiro da sala recebe os pacientes, avalia juntamente com o médico a diálise que o paciente vai fazer durante aquele período, atende a qualquer intercorrência que acontece[...] faz toda a parte da sistematização de enfermagem [...]faz conferência de material junto com a pessoa do almoxarifado [...]. (E2)

Quanto à Meta 3, observaram-se intercorrências durante a hemodiálise relacionadas a medicamentos de alta vigilância ou potencialmente perigosos, que apresentam risco aumentado de provocar danos significativos aos pacientes em decorrência de falha no processo de utilização.

Uma ocorrência evidenciada foi o sangramento relacionado ao uso de heparina no sistema de hemodiálise, bem como coagulação do sistema.

[...] pós-cirurgia ele [o paciente] vem sangrando, aí você tem que avaliar a questão do sangramento para não fazer anticoagulante na diálise, também tem que ver a questão dos exames juntamente com o médico para saber se esse paciente talvez precise de transfusão [...]. (E2)

[...] A intercorrência que acontece mesmo é coagulação do sistema, das linhas, do capilar, o cateter às vezes o fluxo não é bom [...].(TE9)

Outra intercorrência comum com medicamentos de alta vigilância está relacionada à instabilidade hemodinâmica devido ao uso frequente de drogas vasoativas, principalmente nos pacientes internados que se submetem ao procedimento hemodialítico:

[...]às vezes ocorre também hipotensão, hipertensão, hipoglicemia, chegando até o paciente parar, [se estiver em uso] de uma droga vasoativa para aumentar a pressão [...]. (TE10)

Todos os medicamentos eram armazenados em gavetas devidamente identificadas no posto de enfermagem, porém não havia controle de acesso a estas gavetas; ainda não ocorria a rotulação dos medicamentos na unidade e nem de recipientes, medicamentos de alta vigilância já diluídos eram armazenados sem identificação.

Não se realizava a padronização do número de concentrações de medicamentos e nem a lista completa de medicamentos com nomes semelhantes. Identificou-se uma lista de medicamentos de alta vigilância no posto de enfermagem. Porém, esta lista não abrangia os riscos relacionados à administração de cada um destes medicamentos.

Sobre a Meta 4, foi possível observá-la com mais clareza no processo de trabalho da enfermeira que era encarregada das diálises externas, ao se comunicar com a enfermeira do setor de origem do paciente a fim de levantar se ele reunia condições de dialisar. 
A técnica de Enfermagem que ficava no preparo da máquina fazia a programação com o tipo de solução de cada paciente conforme a prescrição médica, tempo de duração da sessão e as perdas de acordo com o paciente. As técnicas de enfermagem recebiam a máquina programada e levavam até a unidade do paciente, o que poderia ocasionar a realização do procedimento no paciente errado, caso não houvesse comunicação adequada entre os setores. Este erro foi relatado por uma enfermeira:

Antes de ligar o paciente [à máquina de hemodiálise], tem que observar se é o paciente que vai ser ligado, se é o setor certo em relação ao leito, que às vezes já aconteceu de o paciente ser ligado errado [...]. (E3)

Por outro lado, ressaltou-se a preocupação em conferir o paciente, procedimento e prescrição certa:

[...] a gente remove a máquina pronta daqui, é feito o teste, é feito todo o programa da diálise na máquina, leva a máquina prontinha para o leito [...] quando a gente chega lá, checa tudo de novo [...]. (TE2)

Era realizada a dupla checagem do procedimento ou da intervenção a ser iniciada, quando o técnico de Enfermagem da unidade de hemodiálise era encaminhado à unidade do paciente internado a ser dialisado e checava prescrição, nome completo do paciente e os parâmetros da máquina, se estavam condizentes com a prescrição médica.

A Meta 5 deve estar presente nos cuidados que a equipe possui antes de ligar o paciente à máquina de hemodiálise, bem como em todos os procedimentos que envolvem o contato direto com o paciente.

Os curativos eram realizados pelo enfermeiro com técnica asséptica e a higienização das mãos também era realizada constantemente pelos profissionais, porém não havia protocolo que padronizasse esse procedimento e não havia o monitoramento do uso de antimicrobianos.

Outro cuidado realizado pela equipe e observado pelas pesquisadoras diz respeito à manipulação do acesso venoso:

[...] tem que observar como está o acesso desse paciente, se ele realmente tem acesso para ser ligado, se o acesso está pérvio, ligar de uma forma asséptica, para evitar infecção [...]. (E3)

Os cuidados de enfermagem com a desinfecção da máquina entre as sessões dialíticas foram destacados na fala de E7, como forma de prevenção de doenças hematológicas

[...]; nós não sabemos a sorologia do paciente das externas, que são pacientes que chegam graves e a gente tem que dialisar, então em todos os pacientes a máquina tem que ser submetida antes e depois da diálise, a desinfecção química. (E7)

Em relação à Meta 6, como a clientela atendida na unidade é de pacientes críticos, sua condição pode levar à ocorrência de quedas.

[...] eles fazem muita cefaleia, decorrente da retirada da ureia, e geralmente chegam hipertensos, aí ficam hipotensos [...] tem aquele mal estar, aquela súbita tontura [...]. (E2)

Não foi identificada a preocupação dos profissionais quanto ao risco de queda dos pacientes. No entanto, o hospital oferecia um ambiente limpo, arejado, bem iluminado, com cadeiras de rodas para pacientes que não tinham condições de andar. Os pacientes acamados eram dialisados nas próprias macas com as grades de proteção elevadas. Mas a meta não estava adequada ao preconizado pela Joint Commission International, pois não havia instituído na unidade um protocolo para prevenção de quedas.

\section{- DISCUSSÃO}

O perfil das entrevistadas assemelha-se a pesquisas na área: população feminina, trabalhando mais de 30 horas semanais, com grande parte apresentando sobrecarga de trabalho ${ }^{(15)}$.

Estudando a aplicabilidade das metas de segurança do paciente na unidade de diálise, evidenciaramse, como principais fatores contribuintes para incidentes, aqueles relacionados às metas 1, 3 e 4 . 
Estudos internacionais mostram que os principais problemas relacionados à segurança do paciente em hemodiálise envolvem quedas, erros de medicação, eventos relacionados a acesso venoso, erros de dialisador e sangramento prolongado ${ }^{(16)}$.

No que diz respeito à Meta 1, preconiza-se que deve existir pelos menos duas formas de identificação do paciente, sendo que estas não devem incluir o uso do número do quarto ou da localização do paciente ${ }^{(12)}$; ainda, a identificação adequada deve ocorrer antes da administração de medicamentos, sangue ou hemocomponentes; antes da coleta de sangue e de outras amostras para exame; e antes da realização de procedimentos e tratamentos.

Já em relação à Meta 2, ressalta-se a necessidade de acompanhamento do paciente pela equipe interdisciplinar, pois o tratamento dialítico exige integração e comunicação com todos os profissionais, para priorizar a qualidade e a segurança ${ }^{(17)}$.

Outro estudo também identificou problemas de comunicação entre provedores de cuidado aos pacientes em serviços de diálise hospitalar e ambulatorial. Os entrevistados relataram que a comunicação não era efetiva e expressaram preocupações de que isso estava afetando negativamente o cuidado dos pacientes e colocando-os em risco de eventos adversos ${ }^{(6)}$; foram também capazes de identificar barreiras à boa comunicação e expressaram desejo de padronização do processo de comunicação na alta, mas não descreveram melhorias específicas ${ }^{(6)}$.

Surgiu como problema a passagem de informações relevantes do paciente por telefone entre as enfermeiras, como condição clínica e estado geral. Preconiza-se que para reduzir os erros de comunicação, no momento em que se faz uma ordem verbal ou por telefone, deve-se certificar que a informação foi compreendida e registrada corretamente por quem a recebeu ${ }^{(12)}$.

Na Meta 3, as entrevistadas manifestaram um conhecimento satisfatório em relação aos riscos da terapia anticoagulante, porém o serviço não se mostrou seguro quanto ao armazenamento e controle dos medicamentos de alta vigilância, que compartilham várias características - índice terapêutico estreito e o risco de dano significativo. Esses eventos adversos podem levar à incapacidade, à necessidade de hospitalização ou à morte. Enfermeiros interceptam $50 \%$ a $86 \%$ dos erros de medicação antes que cheguem ao paciente ${ }^{(18)}$.

Deve-se, portanto, envolver times multidisciplinares; realizar duplas checagens independentes; limitar interrupções e distrações durante a administração da medicação; controlar a carga de trabalho e o dimensionamento de pessoal; e reduzir a confusão em torno dos medicamentos com nome e som do nome semelhantes ${ }^{(18)}$.

Medicações de alta vigilância devem ser identificadas com etiqueta vermelha e enviadas às unidades em sacos plásticos vermelhos. Esses fármacos devem ser mantidos no posto de enfermagem, em gavetas com chave e só devem ser manipulados na farmácia ${ }^{(12)}$.

Nos carros de emergência, tais medicamentos devem estar identificados com etiqueta vermelha e separados dos demais. Antes de sua administração pela equipe de enfermagem, deve ser checado o nome completo do paciente; data de nascimento/registro do paciente; nome do medicamento; dose prescrita; via e horário da administração ${ }^{(12)}$; deve identificar a ocorrência de reações adversas com os pacientes e notificá-las ${ }^{(19)}$.

Referente à Meta 4, a dupla checagem garante a correição do procedimento. Estudo com 1506 pacientes em diálise submetidos a procedimentos cirúrgicos gerais não-emergentes identificou que estes são mais propensos a desenvolver pneumonia, intubação não planejada, dependência do ventilador e necessidade de reoperação do que pacientes que não faziam diálise. A submissão ao tratamento dialítico é considerada importante risco para esses pacientes, além de maior risco de complicações vasculares e morte ${ }^{(20)}$.

Embora as máquinas de diálise raramente sejam causa importante de morbidade, os fatores humanos na interface da máquina e a comunicação não efetiva entre os profissionais são fontes comuns de erro. As principais causas de resultados adversos potencialmente reversíveis incluem erros de medicação, infecções, hipercalemia, erros relacionados com o acesso e quedas de pacientes ${ }^{(21)}$.

A engenharia de fatores humanos e exercícios de simulação têm forte potencial para definir a 
finalidade da equipe clínica, e melhorar os processos de cuidados. As observações dos pacientes e sua participação na redução de erros aumentam a eficácia dos esforços de segurança do paciente ${ }^{(21)}$.

Verifica-se a necessidade de aumentar a vigilância dos profissionais no atendimento às metas de segurança, com especial atenção aos procedimentos invasivos e cirurgias nessa clientela por meio do time out, pausa momentânea, tomada pela equipe, antes da incisão da pele, a fim de confirmar itens essenciais à segurança do cliente ${ }^{(22)}$.

Antes da realização da punção da fístula, é necessário que haja avaliação e escolha do melhor e correto local pelo profissional de enfermagem; isto denota cuidado de segurança com o cliente ${ }^{(23)}$. Recomenda-se que a unidade adote práticas seguras, tais como identificação dos pacientes por meio de pulseiras contendo os nomes destes em letras legíveis, o questionamento do paciente de seu nome completo, a identificação do leito e evitar que pacientes com nomes semelhantes fiquem muito próximos ${ }^{(24)}$.

Quanto à Meta 5, é importante destacar que, em pacientes com doença renal submetidos à hemodiálise, as infecções relacionadas aos acessos vasculares podem causar bacteremia ou perda do acesso, além do alto risco de desenvolver infecções de corrente sanguínea ${ }^{(25)}$.

Essa meta trata da adoção de diretrizes, estratégias para treinamento e educação para a higienização das mãos; medidas para prevenção de infecção de corrente sanguínea relacionada ao cateter venoso central; e monitoramento de microrganismos multirresistentes/uso racional de antimicrobianos ${ }^{(26)}$.

Finalmente, no que se refere à Meta 6,um fato preocupante foi a não avaliação do risco de queda dos pacientes relacionado a um distúrbio metabólico consequência da doença renal, que pode comprometer o sistema musculoesquelético a partir do estágio 3 da doença renal crônica ${ }^{(27)}$.

Recomenda-se o desenvolvimento de programas de educação continuada para a equipe de enfermagem, para promover dicussões na identificação e correção dos erros nos serviços de diálise.

O estudo apresentou como limitações o não acompanhamento das diálises realizadas fora da unidade diálise, porque não foi possível aplicar a observação sistemática e a impossibilidade de observar o processo de trabalho de todos os profissionais de enfermagem.

\section{- CONCLUSÃO}

Observar as Metas Internacionais de Segurança do Paciente é relevante, pois a população espera dos serviços de saúde promoção de saúde, prevenção de doenças, cura e reabilitação, evitando danos resultantes da assistência prestada, como contração de infecções por meio das práticas negligentes.

A unidade de diálise em estudo apresentou pontos a serem melhorados, que envolvem o desenvolvimento de estratégias que minimizem os riscos à segurança dos pacientes em relação à identificação, medicamentos de alta vigilância e procedimentos invasivos, implementando protocolos e capacitando toda a equipe de saúde para atuar com base em práticas seguras e seguindo o que for determinado pela instituição, mas observando também normas e padrões internacionais de segurança.

Algumas metas, como comunicação efetiva, redução do risco de infecção e de lesões de pele decorrente de quedas, apresentaram evidências na prática, ou seja, aplicaram-se ações que estavam em conformidade com o recomendado pela Joint Commission International. Porém, é necessário que tais práticas sejam implementadas em sua totalidade e que os pacientes recebam cuidados de qualidade e segurança por parte de toda a equipe de enfermagem.

A pesquisa trouxe contribuições para o processo de trabalho da equipe de enfermagem, assim como para o serviço de hemodiálise, pois alertou para a necessidade de implementação de protocolos e padronizações para a segurança do paciente e também da equipe. Os resultados alcançados podem servir de incentivos e mesmo de marco para outros serviços de semelhante natureza.

\section{REFERÊNCIAS}


1. Duarte SCM, Stipp MAC, da Silva MM, de Oliveira FT. Adverse events and safety in nursing care. Rev.bras. enferm. [Internet] 2015;68(1) [acesso em 26 nov 2016]. Disponível: http://dx.doi.org/10.1590/0034-7167.2015680120p.

2. Runciman W, Hibbert P, Thomson R, Van Der Schaaf T, Sherman H, Lewalle P. Towards an International Classificationfor Patient Safety: key concepts and terms. Int J Qual Health Care. 2009;21(1):18-26.

3. Sociedade Brasileira de Nefrologia (SBN) SBN informa. Publicação oficial da Sociedade Brasileira de Nefrologia. [Internet] 2016;(106) [acesso em 28 nov 2016]. Disponível:http://sbn.org.br/app/uploads/sbninforma106_2016_ site-1.pdf.

4. Bray BD, Boyd J, Daly C, Doyle A, Donaldson K, Fox JG, et al. How safe is renal replacement therapy? A national study of mortality and adverse events contributing to the death of renal replacement therapy recipients. Nephrol Dial Transplant. 2014;29(3):681-7.

5. Reilly JB, Marcotte LM, Berns JS, Shea JA. Handoff communication between hospital and outpatient dialysis units at patient discharge: a qualitative study. JtComm J Qual Patient Saf. 2013;39(2):70-6.

6. de Sousa MRG, Silva AEBC, Bezerra ALQ, de Freitas JS, Miasso AI. Adverse events in hemodialysis: reports of nursing professionals. Rev. esc. enferm. USP. [Internet] 2013;47(1) [acesso em 27 mai 2013]. Disponível: http:// dx.doi.org/10.1590/S0080-62342013000100010.

7. Frazão CMFQ, Delgado MF, Araújo MGA, Lima e Silva FBB, de Sá JD, Lira ALBC.Nursing care for chronic renal patients on hemodialysis. Rev. Rene. [Internet] 2014;15(4) [acesso em 26 nov 2016]. Disponível: http://dx.doi. org/10.15253/2175-6783.2014000400018.

8. Mendes LMS, Machado FB, Fonseca AS. Perspectivas existentes relacionadas à assistência de enfermagem de um hospital acreditado. Nursing (São Paulo). 2012;14(167):192-6.

9. Ministério da Saúde (BR). Agencia Nacional de Vigilância Sanitária. Segurança do paciente e qualidade em serviços de saúde. Assistência Segura: Uma Reflexão Teórica Aplicada à Prática. [Internet]. Brasília (DF): Ministério da Saúde; 2013 [acesso em 25 nov 2016]. Disponível: http://www20.anvisa.gov.br/segurancadopaciente/images/ documentos/livros/Livro1-Assistencia_Segura.pdf.

10.Ministério da Saúde (BR). Resolução n. 36, de 25 de julho de 2013. Agência Nacional de Vigilância Sanitária. Institui ações para a segurança do paciente em serviços de saúde e dá outras providências. Brasília; 2013.

11. Silva LD. Segurança do paciente no contexto hospitalar. Rev. enferm. UER]. [Internet] 2012;20(3) [acesso em 22 mai 2013].Disponível: http://www.facenf.uerj.br/v20n3/v20n3a01.pdf.

12. Joint Commission International.Padrões de Acreditação da Joint Commission International para Hospitais. $4^{\mathrm{a}}$ ed. Rio de Janeiro; 2011.

13. Minayo MCS. O desafio do conhecimento: pesquisa qualitativa em saúde. 13ª ed. São Paulo:Hucitec; 2013.

14. Ministério da Saúde (BR). Conselho Nacional de Saúde. Diretrizes e normas regulamentadoras de pesquisa envolvendo seres humanos. Resolução n. 466, de 12 de dezembro de 2012. Brasília; 2012.

15. de Magalhães AMM, Dall'Agnol CM, Marck PB.Nursing workload and patient safety - a mixed method study with an ecological restorative approach. Rev. Latino-Am. Enfermagem. [Internet] 2013;21(n.esp) [acesso em 22 mai 2013]. Disponível: http://dx.doi.org/10.1590/S0104-11692013000700019.

16. Garrick R, Kliger A, Stefanchik B. Patient and facility safety in haemodialysis: opportunities and strategies to develop a culture of safety. Clin J Am Soc Nephrol. 2012;7(4):680-8.

17. Santana JCB, Fortes NM, Monteiro CLA, Carvalho IM, Leonardo LMU, de Albuquerque PG. Assistência de enfermagem em um serviço de terapia renal substitutiva: implicações no processo do cuidar. Enferm. Rev. [Internet] 2012;15(2) [acesso em 26 mar 2016]. Disponível: http://periodicos.pucminas.br/index.php/enfermagemrevista/ article/view/4082/4332.

18. Anderson P, Townsend T. Preventing high-alert medication errors in hospital patients. Am Nurs Today. [Internet] 2015;10(5) [acesso em 28 nov 2016]. Disponível: https://www.americannursetoday.com/preventinghigh-alert-medication-errors/. 
19. Beltrame V, Hardt C, Madureira VSF, Dall'Agnol J, da Silva TG. Intervenções de enfermagem nas intercorrências do tratamento hemodialítico. Ágora: R. Divulg. Cient. [Internet] 2011;18(1) [acesso em 26 mar 2016]. Disponível:http://www.periodicos.unc.br/index.php/agora/article/view/252/334.

20. Gajdos C, Hawn MT, Kile D, Robinson, TN, Henderson WG. Risk of major nonemergent inpatient general surgical procedures in patients on longterm dialysis. JAMA Surg. 2013;148(2):137-43.

21. Kliger AS. Maintaining safety in the dialysis facility.Clin J Am Soc Nephrol. [Internet] 2015;10(4) [acesso em 28 nov 2016]. Disponível: http://dx.doi.org/10.2215/CJN.08960914.

22. Grigoleto ARL, Gimenes FRE, Avelar MCQ. Segurança do cliente e as ações frente ao procedimento cirúrgico. Rev. Eletr. Enf. [Internet] 2011;13(2) [acesso em 26 mar 2016]. Disponível: http://dx.doi.org/10.5216/ree.v13i2.10326.

23. Moreira AGM, de Araújo STC, Torchi TS. Preservação da fístula arteriovenosa: ações conjuntas entre enfermagem e cliente. Esc. Anna Nery. [Internet] 2013;17(2) [acesso em 26 mar 2016]. Disponível: http://dx.doi. org/10.1590/S1414-81452013000200008.

24. Tase TH, Lourenção DCA, Bianchini SM, Tronchin DMR. Patient identification in healthcare organizations: an emerging debate. Rev. Gaúcha Enferm. [Internet] 2013;34(3) [acesso em 26 mar 2016]. Disponível: http://dx.doi. org/10.1590/S1983-14472013000300025.

25. Ferreira ACB, Deprá MM, Pies OTC, Sousa ICR, Rocha LKM, Saraiva Filho JCP. Infecções em cateter de hemodiálise: aspectos microbiológicos e de resistência em uma unidade de referência de Belém. Rev. Soc. Bras. Clín. Méd. [Internet] 2014;12(4) [acesso em 26 mar 2016]. Disponível: http://files.bvs.br/upload/S/1679-1010/2014/ v12n4/a4372.pdf.

26. Henrique DM, Tadeu CN, Alves FH, Trindade LPC, Fernandes MSR, Macedo ML, et al. Fatores de risco e recomendações atuais para prevenção de infecção associada a cateteres venosos centrais: uma revisão de literatura. Rev Epidemiol Control Infect. [Internet] 2013;3(4) [acesso em 01 jun 2016]. Disponível: https://online. unisc.br/seer/index.php/epidemiologia/article/view/4040/3252.

27. Galvão MH, Santos LIS, Barcelar JM, Marinho PEM.Evaluación de la capacidad de la Escala Internacional de Eficacia de Caídas en discriminar riesgo de caídas en pacientes con enfermedad renal crónica sometidos a hemodiálisis. Fisioter. Pesqui. [Internet] 2013;20(2) [acesso em 26 mar 2016]. Disponível: http://dx.doi.org/10.1590/ S1809-29502013000200009. 